\title{
High-Throughput Mechanistic Screening of Epigenetic Compounds for the Potential Treatment of Meningiomas
}

\author{
Philip D. Tatman ${ }^{1,2,3,{ }^{\dagger}}$, Tadeusz H. Wroblewski ${ }^{1,4,+}{ }^{+}$, Anthony R. Fringuello ${ }^{1}$, Samuel R. Scherer ${ }^{1,4}$, \\ William B. Foreman ${ }^{1,4}$, Denise M. Damek ${ }^{1,4}$, Kevin Lillehei ${ }^{1}$, A. Samy Youssef ${ }^{1}$, Randy L. Jensen ${ }^{5}$, \\ Michael W. Graner ${ }^{1, *}$ and D. Ryan Ormond ${ }^{1, *}$
}

1 Department of Neurosurgery, Anschutz Medical Campus, University of Colorado, Aurora, CO 80045, USA; philip.tatman@cuanschutz.edu (P.D.T.); tadeusz.wroblewski@cuanschutz.edu (T.H.W.); anthonyfringuello@gmail.com (A.R.F.); samuelrscherer@gmail.com (S.R.S.); will.b.foreman@outlook.com (W.B.F.); DENISE.DAMEK@CUANSCHUTZ.EDU (D.M.D.); KEVIN.LILLEHEI@CUANSCHUTZ.EDU (K.L.); SAMY.YOUSSEF@CUANSCHUTZ.EDU (A.S.Y.)

2 Medical Scientist Training Program, Anschutz Medical Campus, University of Colorado, Aurora, CO 80045, USA

3 Department of Pharmacology, Anschutz Medical Campus, University of Colorado, Aurora, CO 80045, USA

4 Department of Neurology, Anschutz Medical Campus, University of Colorado, Aurora, CO 80045, USA

5 Department of Neurosurgery, Huntsman Cancer Institute, University of Utah, Salt Lake City, UT 84112, USA; Randy.Jensen@hsc.utah.edu

* Correspondence: michael.graner@cuanschutz.edu (M.W.G.); david.ormond@cuanschutz.edu (D.R.O.)

+ These authors have equal contribution.

\section{check for} updates

Citation: Tatman, P.D.; Wroblewski, T.H.; Fringuello, A.R.; Scherer, S.R.;

Foreman, W.B.; Damek, D.M.; Lillehei, K.; Youssef, A.S.; Jensen, R.L.; Graner, M.W.; et al. High-Throughput

Mechanistic Screening of Epigenetic Compounds for the Potential Treatment of Meningiomas. J. Clin. Med. 2021, 10, 3150. https:// doi.org/10.3390/jcm10143150

Academic Editor: Morgan Broggi

Received: 21 May 2021

Accepted: 7 July 2021

Published: 16 July 2021

Publisher's Note: MDPI stays neutral with regard to jurisdictional claims in published maps and institutional affiliations.

Copyright: (c) 2021 by the authors. Licensee MDPI, Basel, Switzerland. This article is an open access article distributed under the terms and conditions of the Creative Commons Attribution (CC BY) license (https:// creativecommons.org/licenses/by/ $4.0 /)$.
Abstract: Background: Meningiomas are the most common primary central nervous system tumors. $20-30 \%$ of these tumors are considered high-grade and associated with poor prognosis and high recurrence rates. Despite the high occurrence of meningiomas, there are no FDA-approved compounds for the treatment of these tumors. Methods: In this study, we screened patient-cultured meningiomas with an epigenetic compound library to identify targetable mechanisms for the potential treatment of these tumors. Meningioma cell cultures were generated directly from surgically resected patient tumors and were cultured on a neural matrix. Cells were treated with a library of compounds meant to target epigenetic functions. Results: Although each tumor displayed a unique compound sensitivity profile, Panobinostat, LAQ824, and HC toxin were broadly effective across most tumors. These three compounds are broad-spectrum Histone Deacetylase (HDAC) inhibitors which target class I, IIa, and IIb HDACs. Panobinostat was identified as the most broadly effective compound, capable of significantly decreasing the average cell viability of the sample cohort, regardless of tumor grade, recurrence, radiation, and patient gender. Conclusions: These findings strongly suggest an important role of HDACs in meningioma biology and as a targetable mechanism. Additional validation studies are necessary to confirm these promising findings, as well to identify an ideal HDAC inhibitor candidate to develop for clinical use.

Keywords: meningioma; HDAC inhibitors; panobinostat; LAQ824; HC toxin; high-throughput screening

\section{Introduction}

Meningiomas comprise $33.8 \%$ of all primary brain tumors, making them the most common primary central nervous system (CNS) tumors [1]. From 20-30\% of meningiomas are classified by the World Health Organization (WHO) as grade II or III meningiomas, which have five-year recurrence rates of 38-55\% and 79\%, respectively [2-5]. While higher-grade meningiomas have poor outcomes overall, it has been previously reported that patients with grade I meningiomas also experience long-term neurological deficits and reduced overall survival [6]. These tumors lack a United States Food and Drug Administration (FDA)-approved pharmacotherapeutic agent [7]. Surgery remains the primary treatment modality for accessible, symptomatic meningiomas, often followed by radiation therapy. 
Treatment options for recurrent meningiomas after maximally feasible surgery and radiation are limited, with minimal efficacy shown to date from chemotherapy $[7,8]$. The paucity of treatment options and poor outcomes for patients with meningiomas necessitate additional research into targetable mechanisms for the development of novel therapies.

Previous work on meningioma genetics has yielded little clinical benefit, despite significant findings. NF2 (neurofibromatosis 2) is the most common mutation in meningiomas, occurring in $40-60 \%$ of sporadic meningiomas, and has been implicated as a driver of meningioma tumorigenesis [9,10]. Two independent studies have identified TRAF7 (TNF receptor-associated factor 7), AKT1 (v-akt murine thymoma viral oncogene homolog 1), and $S M O$ (Smoothened, frizzled family receptor) mutations as drivers of meningioma oncogenesis [11,12]. Mutations in TRAF7 have been identified in nearly one-fourth of meningiomas, while mutations in $A K T 1$ and $S M O$ have lower prevalence and are associated with higher grade NF2 wild-type tumors [12]. POLR2A (RNA Polymerase II Subunit A) mutations in meningiomas have also been identified, being linked to a meningothelial histology and tuberculum sellae tumors [13]. More recently, mutations in TERT (telomerase reverse transcriptase) and CDKN2A (cyclin-dependent kinase inhibitor 2A) have been shown to be potential prognostic indicators in higher grade meningiomas [14,15]. Mutations in the TERT promoter have been associated with worse prognosis and decreased survival rates in grade II and III meningiomas, and $C D K N 2 A$ mutation or gene deletion has been associated with meningioma recurrence [15-18]. Although the mutational landscape of meningiomas is becoming more well defined, these findings have not yet led to additional, or improved, therapies.

A plausible solution to the lack of pharmacotherapies for the treatment of meningiomas is to directly measure their drug sensitivities. Screening meningiomas against a compound library enables direct insight into targetable mechanisms as well as potential candidate compounds, depending on library construction. With respect to compound library identification, a promising class of mechanisms with the potential to treat meningiomas is epigenetics. Epigenetics are defined as all processes that regulate structure and access to DNA resulting in transcriptional changes, DNA replication, and DNA repair [19-21]. These are described as "writers" (which add chromatin and histone modifications), "erasers" (which remove modifications), and "readers" (which recognize modifications and mediate effects) of epigenetics [22]. Previous studies have identified epigenetic dysregulation as a driver of meningioma oncogenesis and recurrence [23-26]. CpG island and promoter DNA methylation profiles are known to vary with meningioma pathophysiology and clinical outcomes [24-27]. Furthermore, meningioma whole-genome DNA methylation profiles correlate more accurately to clinical prognosis, compared with the current WHO tumor grading system. Additionally, histone deacetylases (HDACs) have been implicated in meningiomas through the regulation of transcriptional changes, downstream of PI3K/Akt, in the setting of NF2 deletion $[9,28-30]$. The connection between epigenetic dysregulation of meningiomas and clinical outcomes warrants a comprehensive investigation into the targeting of epigenetic mechanisms as a viable treatment avenue for meningiomas.

To identify targetable epigenetic mechanisms for the treatment of meningiomas, we performed a high throughput drug screen of 32 patient-cultured meningiomas against a 139-compound epigenetic library. The compounds in the library were designed to modulate activities of various classes of epigenetic effectors as a mechanistic-based screen. The cultured meningiomas were screened within 3 weeks of surgical resection, establishing a rapid means to identify potential drug sensitivities for therapeutic exploitation. Among the different classes of targets, multiple drugs that target HDACs were identified as having the ability to significantly inhibit meningioma growth, suggesting that HDAC inhibition is a promising therapeutic modality for the treatment of meningiomas.

\section{Materials and Methods}

\subsection{Patient Identification, Demographics, and Tumor Collection}

Patients undergoing surgery for meningiomas were identified through the Neurosurgery Nervous System Biorepository at the University of Colorado, Anschutz Medical 
Campus. From a period of September 2018 to May of 2019, tumor specimens were collected at the time of surgery and transported directly to our lab for tissue culture. In accordance with IRB protocol (IRB \#13-3007), patients were consented to that protocol, and were deidentified. Established patient-derived meningioma cell lines, provided by Dr. Randy Jensen from the University of Utah (IRB \#00010924), were also included in this study. For each tumor, we recorded relevant demographic information including patient age and gender, as well as tumor characteristics including grade, histologic subtype, primary vs. recurrent, and history of radiation and chemotherapy.

\subsection{Tissue Culture}

Meningiomas were collected from the operating rooms at the University of Colorado Anschutz Medical Campus and sectioned into $1 \mathrm{~mm} \times 1 \mathrm{~mm}$ pieces in sterile PBS (pH 7.4), under sterile tissue culture hood conditions. The tumors were then digested with dispase into single-cell suspensions and plated in flasks containing a thin film of decellularized bovine neural matrix [31]. Meningiomas were cultured in high glucose DMEM with 15\% FBS, 1\% pen/strep, and 1\% Glutamax and passaged weekly until used. All tumors were screened within three weeks of surgical resection and never cryogenically frozen, with the exception of tumors originating from the University of Utah.

\subsection{Epigenetic Compound Panel Screening}

Epigenetic compound screening was completed with a 139-compound epigenetic library (Cayman Chemical, Ann Arbor, MI, USA). Cells were plated into neural-matrix coated 96-well plates and seeded at a density of 2500 cells per well. After $24 \mathrm{~h}$ in culture, individual epigenetic compounds were added to individual wells to a final concentration of $1 \mu \mathrm{M}$. Seventy-two hours after the addition of the library, cell viability was determined via MTS assay. Each compound was tested in triplicate for each tumor.

\subsection{Cell Viability}

The cell viability assay was performed with a MTS tetrazolium assay, as previously described [32]. The MTS reagent was prepared with PBS (pH 7.4), to which MTS powder $(2 \mathrm{mg} / \mathrm{mL})$ and PES powder $(0.21 \mathrm{mg} / \mathrm{mL})$ were added. The MTS reagent was added to each well for a final MTS concentration of $0.33 \mathrm{mg} / \mathrm{mL}$. Absorbance was recorded at $490 \mathrm{~nm}$ in a Biotek plate reader (BioTek, Winooski, VT, USA).

\subsection{Statistics}

All statistics were calculated in the $R$ statistical suite (version 4.0.2) (https://wwww.r-project. $\mathrm{org} /$ ). All plates were background subtracted and normalized to vehicle controls, where the vehicle control was comprised of $0.01 \%$ DMSO in media. Significantly effective compounds for individual tumors were identified by filtering for $p$ values less than 0.05 , calculated using the Mann-Whitney U test (confidence level of 0.95). Broadly effective compounds across the entire cohort, or given subgroup of tumors, were identified using the Student's $t$ test (confidence level of 0.95 ) with significance determined by a $p$ value less than 0.05 . To determine the significance between three or more subgroups of tumors, an ANOVA with a Tukey HSD post-hoc test (confidence level of 0.95 ) was conducted with a $p$ value of less than 0.05. Data are displayed as the mean $+/-$ standard deviation.

\section{Results}

\subsection{Patient Demographics and Tumor Information}

Thirty-two tumors were cultured from patients, four of which were from Utah and are indicated as such with an asterisk in Table 1. The mean age of patients in our cohort at the time of surgery was $57+/-12.5$ SD years, ranging from 38-95 years (Table 1). A total of $21.9 \%$ of the patients were male $(n=7)$ and $78.1 \%$ female $(n=25$, Table 1$)$. The cohort of tumors consisted of 25 grade I tumors, 6 grade II tumors, and 1 grade III tumor, 
including 3 recurrent and 29 primary tumors (Table 1). The tumors were further separated by histologic subtype, history of radiation, and chemotherapy as described in Table 1.

Table 1. Patient demographics for the meningioma cohort.

\begin{tabular}{|c|c|c|c|c|c|c|c|c|}
\hline No. & Sample & Age & Gender & Grade & Histologic Subtype & Primary/Recurrent & Radiation & Chemotherapy \\
\hline 1 & *IOMM Lee & 61 & Male & 3 & Anaplastic & Recurrent & Unknown & Unknown \\
\hline 2 & ${ }^{*} \mathrm{CH} 157$ & 59 & Female & 2 & Unknown & Primary & Unknown & Unknown \\
\hline 3 & $\mathrm{~J} 8-4$ & 48 & Female & 2 & Atypical & Primary & No & No \\
\hline 4 & $\mathrm{~J} 13-2$ & 54 & Female & 2 & Atypical & Primary & No & No \\
\hline 5 & $\mathrm{~J} 17-2$ & 68 & Female & 2 & Atypical & Primary & No & No \\
\hline 6 & J19-3 & 95 & Female & 2 & Atypical & Primary & No & No \\
\hline 7 & $\mathrm{~J} 19-4$ & 49 & Female & 2 & Atypical & Recurrent & No & No \\
\hline 8 & ${ }^{*} \mathrm{GAR}$ & 71 & Male & 1 & Unknown & Primary & Unknown & No \\
\hline 9 & $\mathrm{H} 23-3$ & 83 & Female & 1 & $\begin{array}{c}\text { Small cell with } \\
\text { psammoma bodies }\end{array}$ & Primary & No & No \\
\hline 10 & $\mathrm{~J} 10-6$ & 54 & Male & 1 & Meningothelial & Primary & No & No \\
\hline 11 & $\mathrm{~J} 11-2$ & 57 & Female & 1 & Not Specified & Primary & No & No \\
\hline 12 & J11-6 & 56 & Female & 1 & Not Specified & Primary & No & No \\
\hline 13 & $\mathrm{~J} 11-7$ & 58 & Male & 1 & Meningothelial & Primary & No & No \\
\hline 14 & $\mathrm{~J} 15-4$ & 40 & Female & 1 & Myxoid & Primary & No & No \\
\hline 15 & $\mathrm{~J} 16-5$ & 49 & Female & 1 & Psammomatous & Primary & No & No \\
\hline 16 & $\mathrm{~J} 17-7$ & 49 & Female & 1 & Not Specified & Primary & No & No \\
\hline 17 & $\mathrm{~J} 18-6$ & 64 & Female & 1 & Meningothelial & Primary & $\begin{array}{l}\text { Gamma } \\
\text { Knife 36Gy }\end{array}$ & $\begin{array}{l}6 \text { Cycles of TAC } \\
\text { Chemotherapy }\end{array}$ \\
\hline 18 & $\mathrm{~J} 18-7$ & 41 & Female & 1 & Meningothelial & Primary & No & No \\
\hline 19 & $\mathrm{~J} 19-2$ & 44 & Female & 1 & Psammomatous & Primary & No & No \\
\hline 20 & $\mathrm{~J} 19-5$ & 39 & Female & 1 & Meningothelial & Primary & No & No \\
\hline 21 & J19-6 & 44 & Female & 1 & Transitional & Primary & No & No \\
\hline 22 & $\mathrm{~J} 19-7$ & 57 & Female & 1 & Meningothelial & Primary & No & No \\
\hline 23 & $\mathrm{~J} 2-2$ & 65 & Female & 1 & Meningothelial & Primary & No & No \\
\hline 24 & J21-4 & 55 & Female & 1 & $\begin{array}{c}\text { Rhabdoid } \\
\text { Morphology Present }\end{array}$ & Primary & No & No \\
\hline 25 & $\mathrm{~J} 21-5$ & 65 & Male & 1 & Secretory & Primary & No & No \\
\hline 26 & $\mathrm{~J} 21-7$ & 51 & Female & 1 & Fibrous & Recurrent & No & No \\
\hline 27 & J4-1 & 63 & Female & 1 & Fibrous & Primary & No & No \\
\hline 28 & J4-4 & 67 & Male & 1 & Fibrous & Primary & No & No \\
\hline 29 & $\mathrm{~J} 7-6$ & 57 & Female & 1 & Transitional & Primary & No & No \\
\hline 30 & J8-3 & 38 & Female & 1 & Meningothelial & Primary & No & No \\
\hline 31 & J9-3 & 70 & Female & 1 & Psammomatous & Primary & No & No \\
\hline 32 & ${ }^{*} \mathrm{JEN}$ & 55 & Male & 1 & Psammomatous & Primary & Unknown & No \\
\hline
\end{tabular}

Tumor sample identifier code with patient age and gender as well as tumor classification variables including histologic subtype, whether the tumor is primary or recurrent, and history of radiation and chemotherapy. University of Utah cell lines are denoted by an asterisk. TAC chemotherapy $=$ Taxotere, Adriamycin, and Cyclophosphamide.

\subsection{Meningiomas Are Broadly Sensitive to Epigenetic Inhibition and Have Unique Drug Sensitivity Profiles}

Screening our cohort of patient-cultured meningiomas demonstrated that epigenetic compounds can significantly decrease cell viability (Figure 1a). On average, $27+/-15.3 \mathrm{SD}$ compounds significantly reduced cell viability per tumor (Supplementary Tables S1 and S2). Across all tumors, cell viability was reduced from $100 \%$ to $75 \%$ in $29 / 32$ tumors, to $50 \%$ in 
24/32 tumors, and to 25\% in 3/32 tumors (Figure 1a, Supplementary Tables S1 and S2), clearly showing that meningiomas can be inhibited by epigenetic compounds.

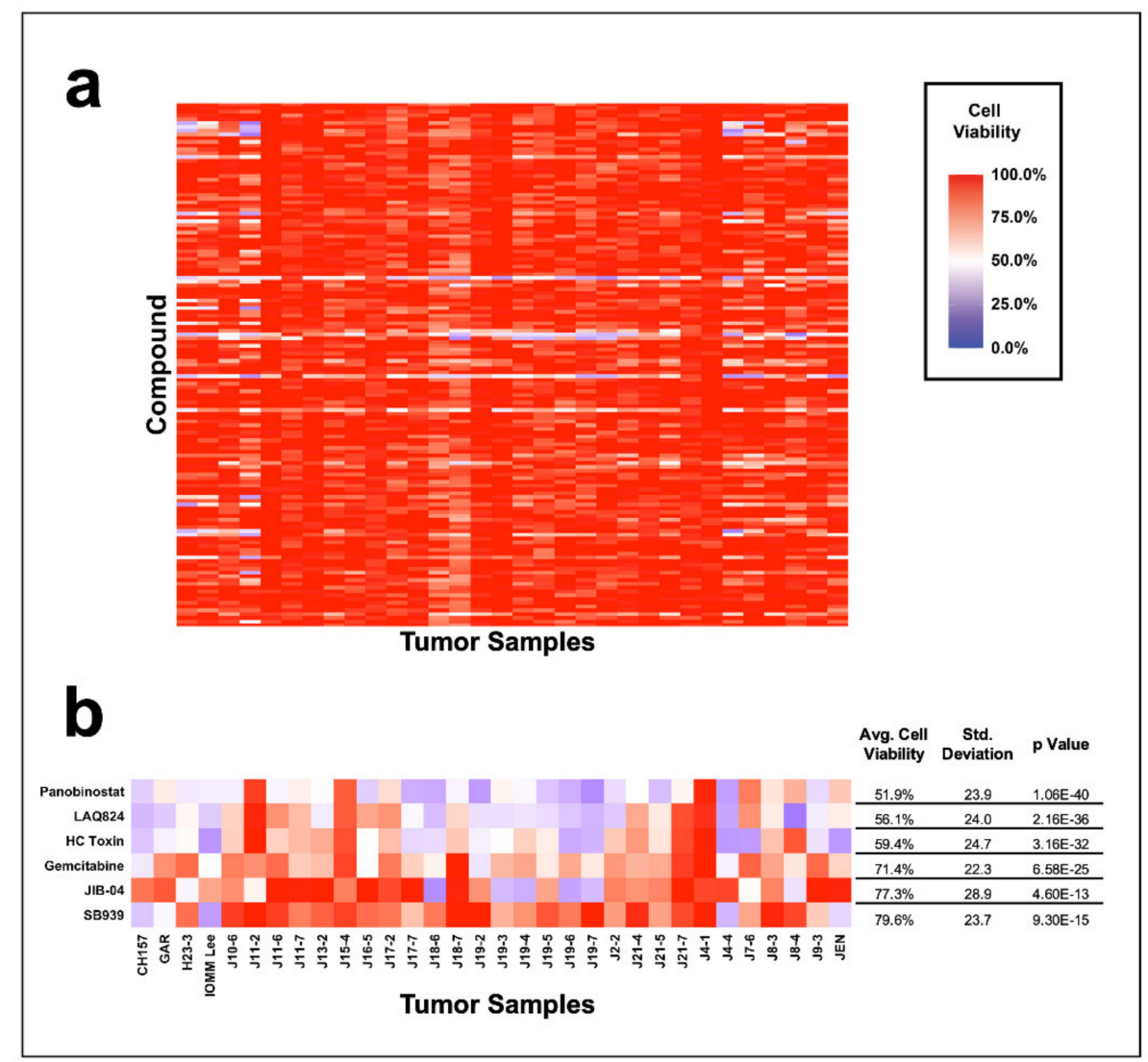

Figure 1. Meningioma sensitivity to the epigenetic compound library. (a) Heat map of meningiomas screened against 139-compound epigenetic panel. (b) Heat map of broadly effective compounds, identified as compounds that significantly decrease the average cell viability of the cohort to $80 \%$ or lower and have a $p$ value less than 0.05 .

Broadly effective compounds were identified as those that reduced cell viability to an average of $80 \%$, or less, across our tumor cohort (not necessarily individual tumors), as well as a $p$ value of less than 0.05 (Figure $1 \mathrm{~b}$, Supplementary Table S3). This screen identified panobinostat $\left(51.9 \%+/-23.9 \mathrm{SD}, p=1.06 \times 10^{-40}\right)$; LAQ824 $\left(56.1 \%+/-24 \mathrm{SD}, p=2.16 \times 10^{-36}\right)$; HC Toxin $\left(59.4 \%+/-24.7 \mathrm{SD}, p=3.16 \times 10^{-32}\right)$; gemcitabine $(71.4 \%+/-22.3 \mathrm{SD}, p=6.58$ $\left.\times 10^{-25}\right)$; JIB-04 $\left(77.3 \%+/-28.9 \mathrm{SD}, p=4.60 \times 10^{-13}\right)$; and SB939 $(79.6 \%+/-23.7 \mathrm{SD}$, $p=9.30 \times 10^{-15}$ ). HC Toxin significantly reduced cell viability in $27 / 32$ tumors, panobinostat reduced cell viability in 26/32 tumors, and LAQ824 and gemcitabine reduced cell viability in 25/32 tumors (Figure 1b). Only one tumor out of this cohort did not have a significant reduction in cell viability after epigenetic inhibition (Supplementary Table S4). These data demonstrate the common biology among meningiomas based on their sensitivity to a small cohort of drugs, as well as the potential to target a common mechanism in meningiomas.

To identify compounds specific to each tumor, we rank ordered compounds that significantly reduced cell viability by a $p$ value less than 0.05 and displayed the top five for each tumor (Figure 2, Supplementary Table S4). This analysis highlights the most effective compounds per individual tumor. Interestingly, panobinostat was the most effective single agent in $44 \%(14 / 32)$ of tumors, followed by JIB-04 in 19\% (6/32), LAQ824 in 13\% (4/32), HC Toxin in 6\% (2/32), OTX015 in 6\% (2/32), and UNC0631 in 6\% (2/32). Despite meningiomas having unique drug sensitivity profiles (Figure 1a), they share a high degree of sensitivity to a small group of compounds (Figure 2). Unsurprisingly, of the broadly effective compounds reported in the previous paragraph, panobinostat appeared in $75 \%$ 
$(24 / 32)$ of the top five most effective compounds per tumor, LAQ824 in $72 \%(23 / 32)$, HC Toxin in 66\% (21/32), gemcitabine in 34\% (11/32), JIB-04 in 34\% (11/32), and SB939 in 9\% $(3 / 32)$. While the unique sensitivity profiles of individual meningiomas suggest that a personalized approach to treatment may be most efficacious (Figures 1a and 2), this analysis shows that most meningiomas in our screen are sensitive to a small number of compounds.

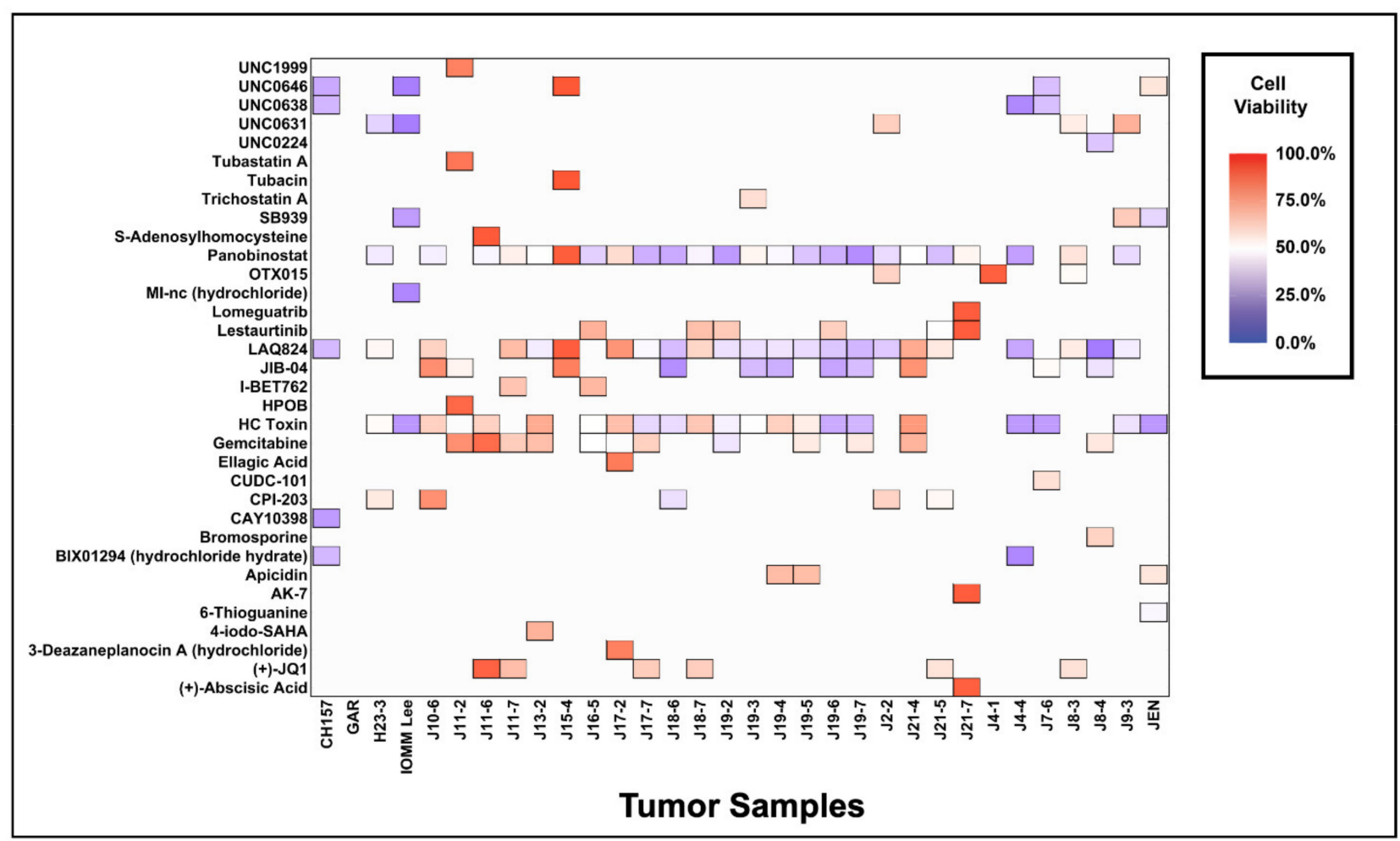

Figure 2. Top five most effective epigenetic compounds per tumor. Heat map of the most effective compounds, determined as the top five compounds that reduce cell viability the most for each tumor. The top five compounds are displayed as filled tiles in the heat map, while compounds that were not in the top five remain as white space.

\subsection{Influence of Grade on the Use of Epigenetic Compounds for the Treatment of Meningiomas}

We next examined the differences in compound sensitivity with respect to tumor grade (Supplementary Figure S1). The most broadly effective compound for grade I tumors was panobinostat, with an average cell viability of $51.9 \%+/-24.8 \mathrm{SD}\left(p=1.05 \times 10^{-30}\right.$, Figure 3a, Supplementary Table S5a), and, for grade II tumors, the most broadly effective compound was LAQ824, with an average cell viability of $44.8 \%+/-16.8 \mathrm{SD}$ $\left(p=1.67 \times 10^{-12}\right)$, followed by panobinostat with $52.7 \%+/-19.1 \mathrm{SD}\left(p=3.04 \times 10^{-10}\right.$, Figure 3b, Supplementary Table S5b). LAQ824 was significantly more effective in grade II compared with grade I tumors $(p=0.0228)$, and there were no significant differences between the effectiveness of panobinostat comparing grade I and grade II tumors (Supplementary Table S6). Panobinostat, LAQ824, HC Toxin, gemcitabine, and JIB-04 were all significantly effective at reducing the average cell viability in both grade I and grade II tumors (Figure 3a,b, Supplementary Table S5a,b). Our single grade III tumor demonstrated unique sensitivities with the most effective compounds including UNC0631 $(22.1 \%+/-1.2$ SD, $\left.p=1.82 \times 10^{-35}\right)$; UNC0646 $\left(22.4 \%+/-2.9 \mathrm{SD}, p=1.39 \times 10^{-8}\right)$; MI-NC hydrochloride $\left(24.2 \%+/-7.4 \mathrm{SD}, p=1.33 \times 10^{-4}\right)$; HC Toxin $\left(27.6 \%+/-1.0 \mathrm{SD}, p=1.09 \times 10^{-27}\right)$; and SB939 $\left(28.9 \%+/-4.2\right.$ SD, $p=3.50 \times 10^{-6}$, Figure 3c, Supplementary Table S5c). Notably, the grade III tumor displayed significant sensitivity to many of the compounds found to be efficacious across all meningiomas, including panobinostat resulting in $46.6 \%+/-33.5 \mathrm{SD}$ average cell viability $(p=0.0496)$ and LAQ824 with $39.1 \%+/-31.7$ SD average cell viability $(p=0.0125)$. These findings implicate that each grade of meningioma has a different 
sensitivity profile, though all three grades remain sensitive to a small cohort of broadly effective compounds.

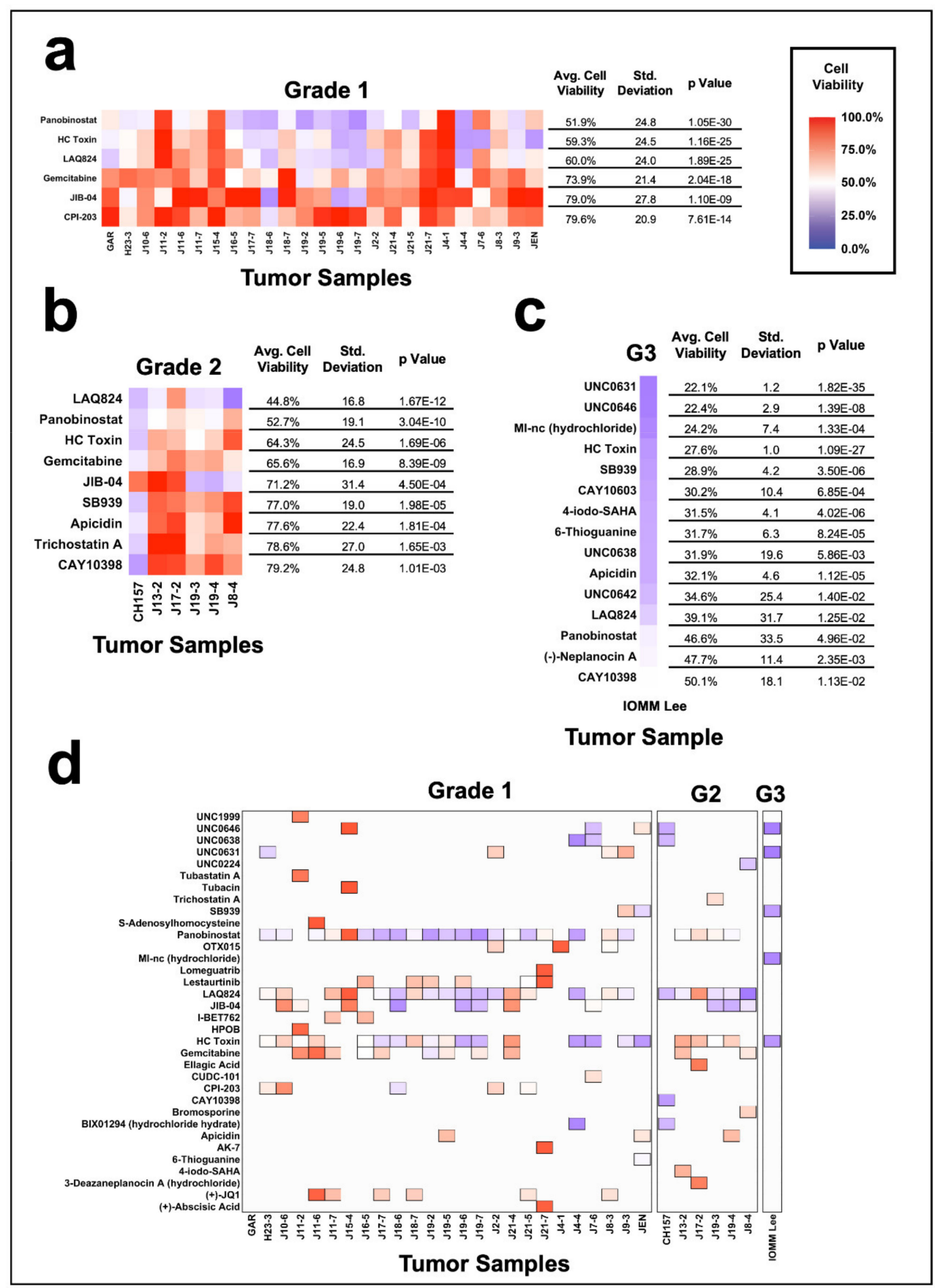

Figure 3. Comparison of the meningioma cohort by tumor grade. Heat maps displaying the broadly most effective compound separated by tumor grade: (a) grade 1 tumors, (b) grade 2 tumors, and (c) grade 3 tumors, denoted as "G3". Broadly effective compounds for each grade are identified as compounds that reduce the average cell viability of the group to $80 \%$ or less and have a $p$ value of less than 0.05 . Heat maps were limited to the top 15 most effective compounds when applicable. (d) Heat map of the top five most effective epigenetic compounds, faceted by grade 1, grade 2 (denoted as "G2"), and grade 3 (denoted as "G3") meningiomas. The top five compounds are displayed as filled tiles in the heat map, while compounds that were not in the top five remain as white space. 
To identify the individual sensitivities of each tumor in the respective grades, we repeated the top most effective compound analysis (Figure 3d). Panobinostat was the most effective compound in 52\% (13/25) of grade I tumors, followed by JIB-04 in 16\% (4/25). For grade II tumors, JIB-04 and LAQ824 were each the most effective compound in 33\% of tumors, inhibiting 2/6 different tumors. UNC0631 was the most effective compound for the grade III tumor.

\subsection{Efficacy of Epigenetic Compounds on Primary vs. Recurrent Meningiomas}

We next sought to understand the compound sensitivity differences between primary and recurrent tumors and found that the effectiveness of individual compounds is largely preserved across both etiologies (Figure 4a,b, Supplementary Figure S2, Supplementary Table S7). Panobinostat was the most broadly effective compound for both primary and recurrent meningiomas resulting in an average cell viability of $52.1 \%+/-24.3 \mathrm{SD}(p=2.34$ $\left.\times 10^{-36}\right)$ for primary tumors and $49.0 \%+/-20.4$ SD in recurrent tumors $\left(p=2.28 \times 10^{-5}\right)$. Primary tumors also exhibited sensitivities to LAQ824 (56.2\% +/ - 23.2 SD, $p=6.62 \times$ $\left.10^{-34}\right)$, HC Toxin $\left(59.2 \%+/-24.3 \mathrm{SD}, p=2.88 \times 10^{-30}\right)$, gemcitabine $(71.5 \%+/-21.0 \mathrm{SD}$, $\left.p=2.11 \times 10^{-24}\right)$, and JIB-04 $\left(78.2 \%+/-28.1 \mathrm{SD}, p=1.09 \times 10^{-11}\right)$; recurrent tumors were broadly sensitive to LAQ824 $\left(55.0 \%+/-31.5 \mathrm{SD}, p=7.91 \times 10^{-4}\right)$, HC Toxin $\left(60.9 \%+/-30.2 \mathrm{SD}, p=4.64 \times 10^{-3}\right)$, apicidin $\left(61.9 \%+/-28.9 \mathrm{SD}, p=2.41 \times 10^{-3}\right)$, and SB939 $\left(62.7 \%+/-29.9 \mathrm{SD}, p=3.37 \times 10^{-3}\right)$. Notably, no significant difference in effectiveness between these compounds was found between primary and recurrent tumors (Supplementary Table S8), suggesting the potential to treat these two etiologies by targeting similar mechanisms.

The five most effective compounds for either primary or recurrent tumors were also identified (Figure 4c). Panobinostat was the number one most effective compound for $45 \%$ $(13 / 29)$ of primary tumors and 33\% (1/3) of recurrent tumors; JIB-04 was the most effective compound for $17 \%(5 / 29)$ primary tumors and 33\% (1/3) of recurrent tumors; and LAQ824 was the most effective compound in $14 \%$ (4/29) of primary tumors. These data suggest that regardless of tumor recurrence, a small cohort of compounds are effective in most meningiomas.

\subsection{Meningioma Sensitivity to Epigenetic Inhibition Based on Radiation History}

To identify the effect of prior radiation on meningioma sensitivity to epigenetic inhibition, we grouped our cohort by tumors with a prior history of radiation, no radiation, or not specified (Supplementary Figure S3). Broadly effective compounds were determined, as described above, and identified for each of the radiation history categories (Figure 5a-c, Supplementary Table S9a-c). Tumors with no history of radiation were broadly sensitive to panobinostat $\left(52.5 \%+/-23.1 \mathrm{SD}, p=7.82 \times 10^{-35}\right)$, LAQ824 $(59.0 \%+/-23.0 \mathrm{SD}, p=6.51$ $\left.\times 10^{-30}\right)$, HC Toxin $\left(62.7 \%+/-24.6 \mathrm{SD}, p=9.51 \times 10^{-26}\right)$, gemcitabine $(73.8 \%+/-18.7 \mathrm{SD}$, $\left.p=1.38 \times 10^{-23}\right)$, and JIB-04 $\left(77.7 \%+/-28.6 \mathrm{SD}, p=4.57 \times 10^{-11}\right)$. The single tumor with prior radiation, which notably was not radiation induced, was significantly inhibited by JIB-04 $\left(25.7 \%+/-9.6 \mathrm{SD}, p=4.3 \times 10^{-3}\right)$, panobinostat $(31.8 \%+/-1.4 \mathrm{SD}, p=2.81$ $\left.\times 10^{-16}\right)$, LAQ824 $\left(35.8 \%+/-8.5 \mathrm{SD}, p=4.18 \times 10^{-3}\right)$, HC toxin $(42.2 \%+/-8.4 \mathrm{SD}$, $\left.p=5.22 \times 10^{-3}\right)$, and CPI-203 $\left(43.5 \%+/-1.3 \mathrm{SD}, p=7.50 \times 10^{-18}\right)$. The radiation nonspecified tumors were sensitive to HC toxin $\left(35.7 \%+/-9.2 \mathrm{SD}, p=3.07 \times 10^{-13}\right)$, SB939 $\left(39.0 \%+/-11.3 \mathrm{SD}, p=6.2 \times 10^{-14}\right)$, UNC0646 $\left(40.5 \%+/-27.1 \mathrm{SD}, p=1.22 \times 10^{-6}\right)$, and LAQ824 $\left(41.5 \%+/-26 \mathrm{SD}, p=8.74 \times 10^{-7}\right)$; those tumors were also significantly inhibited by previously mentioned broadly effective compounds, panobinostat $(52.2 \%+/-30.1 \mathrm{SD}$, $\left.p=2.20 \times 10^{-5}\right)$ and gemcitabine $\left(59.5 \%+/-37.7 \mathrm{SD}, p=1.47 \times 10^{-3}\right)$. 


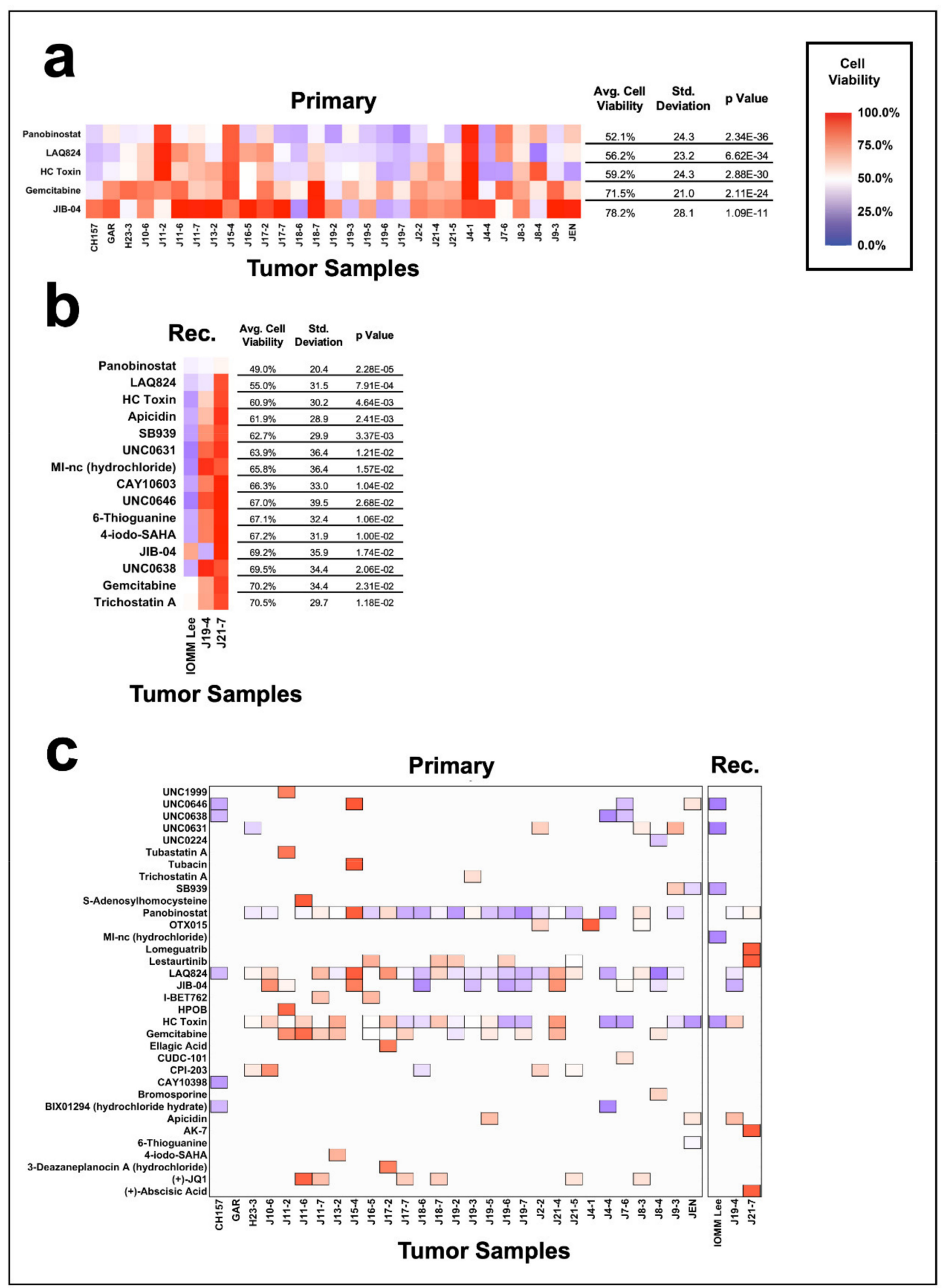

Figure 4. Comparison of the meningioma cohort separated by primary and recurrent tumors. Heat maps displaying the broadly effective compounds for (a) primary meningiomas and (b) recurrent (denoted as "Rec") meningiomas. Broadly effective compounds for primary and recurrent meningiomas are identified as compounds that reduce the average cell viability of the group to $80 \%$ or less and have a $p$ value of less than 0.05 . Heat maps were limited to the top 15 most effective compounds when applicable. (c) Heat map of the top five most effective epigenetic compounds, separated by primary and recurrent (denoted as "Rec.") meningiomas. The top five compounds are displayed as filled tiles in the heat map, while compounds that were not in the top five remain as white space. 


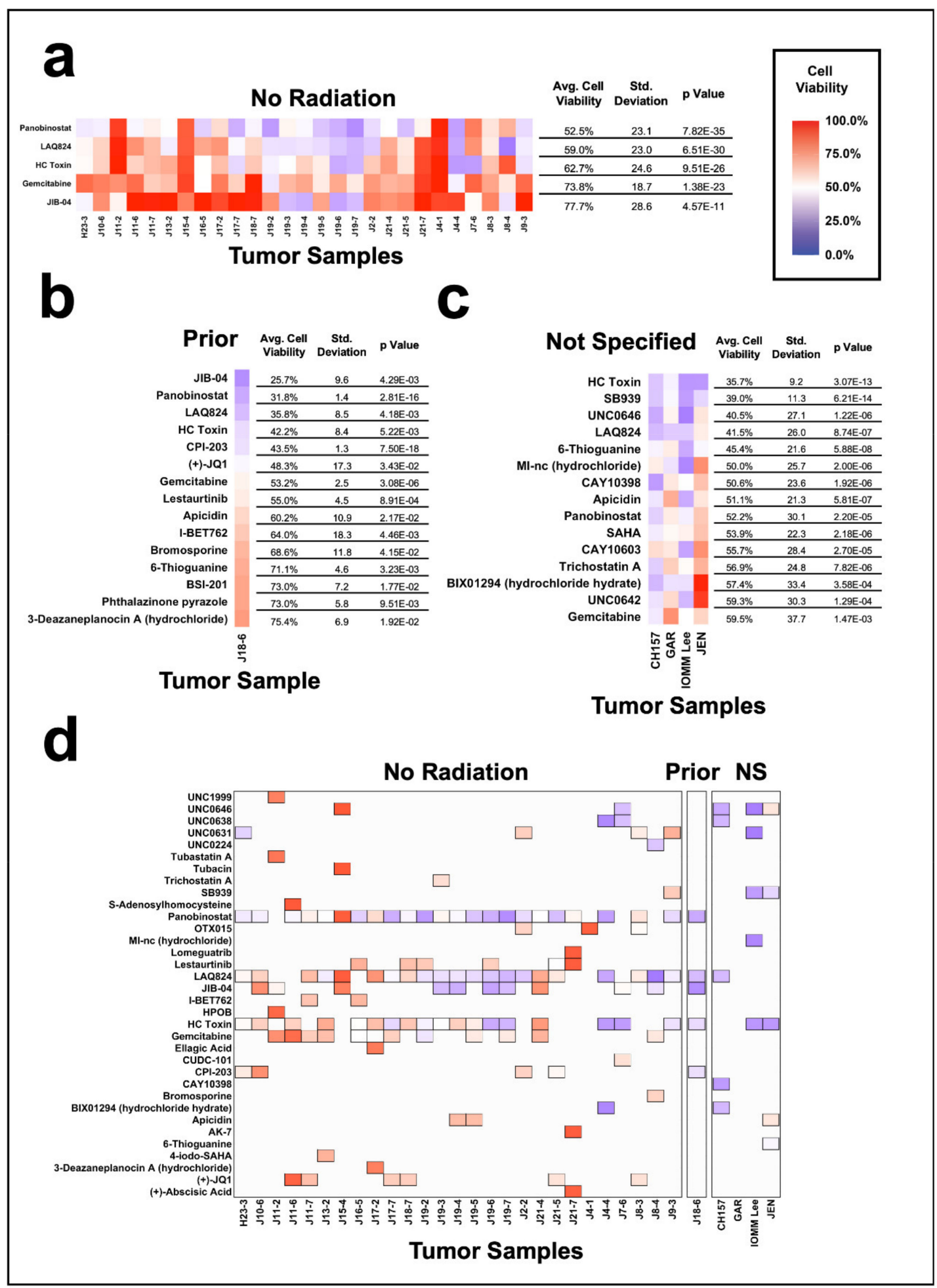

Figure 5. Comparison of the meningioma cohort by history of tumor radiation. Heat maps displaying the broadly most effective compounds for meningiomas with (a) no history of radiation, (b) prior history of radiation (denoted as "Prior"), and (c) tumors with a non-specified history of radiation. Broadly effective compounds for tumors with no radiation, prior radiation, or non-specified radiation are identified as compounds that reduce the average cell viability of the group to $80 \%$ or less and have a $p$ value of less than 0.05 . Heat maps were limited to the top 15 most effective compounds when applicable. (d) Heat map of the top five most effective epigenetic compounds, facetted by meningiomas with no radiation, non-specified history of radiation (denoted as "NS"), and tumors with prior radiation (denoted as "Prior"). The top five compounds are displayed as filled tiles in the heat map, while compounds that were not in the top five remain as white space. 
When analyzing the most effective individual compounds per tumor, grouped by radiation history (Figure 5d, Supplementary Table S9a-c), we found in tumors with no prior history of radiation that panobinostat was the most effective compound in $51.9 \%$ $(14 / 27)$ of tumors, followed by JIB-04 in 18.5\% (5/27) of tumors, and LAQ824 in $11.1 \%$ $(3 / 27)$ of tumors. In our single tumor with prior radiation, the most effective compound was JIB-04. Finally, in our cohort of four tumors with non-specified radiation history, each tumor responded best to a different compound, which included LAQ824, HC Toxin, UNC0631, and CAY-10398.

\subsection{Impact of Gender on Epigenetic Inhibition of Meningiomas}

We next compared the sensitivity of meningiomas to epigenetic inhibition between tumors from female and male patients (Supplementary Figure S4). The most effective compound for female-derived patient tumors was panobinostat with an average cell viability of $53.1 \%+/-23.7 \mathrm{SD}\left(p=4.10 \times 10^{-31}\right)$, followed by LAQ824 $(58.4 \%+/-23.9 \mathrm{SD}, p=3.75$ $\left.\times 10^{-27}\right)$, and HC toxin $\left(62.8 \%+/-24.8 \mathrm{SD}, p=1.10 \times 10^{-23}\right.$, Figure 6a, Supplementary Table S10a). HC toxin was the most broadly effective compound in male-derived tumors, demonstrating an average cell viability of $45.3 \%+/-19.3 \mathrm{SD}\left(p=1.14 \times 10^{-11}\right)$, followed by panobinostat $\left(47.6 \%,+/-24.8 \mathrm{SD}, p=9.62 \times 10^{-11}\right)$ and LAQ824 $(48.1 \%+/-23.2 \mathrm{SD}$, $p=7.08 \times 10^{-11}$, Figure $6 \mathrm{~b}$, Supplementary Table S10b). Comparing the top five compounds from the tumors originating from male and female patients, there was no significant difference in the efficacy of panobinostat, LAQ824, and gemcitabine (Supplementary Table S11). JIB-04 was significantly more effective in female patients than male patients $(p=0.0279)$, while HC Toxin $\left(p=1.19 \times 10^{-3}\right), \operatorname{SB939}\left(p=3.20 \times 10^{-6}\right)$, and 6-thioguanine $\left(p=9.56 \times 10^{-6}\right)$ were significantly more effective in male patients than female patients (Supplementary Table S11).

We then assessed the most effective compound per tumor, separated by gender (Figure 6c). Panobinostat was the most effective compound in $44 \%(11 / 25)$ of tumors from female patients, followed by JIB-04 in $24 \%(6 / 25)$ of tumors, and LAQ824 in $12 \%$ $(3 / 25)$ of tumors. Panobinostat was also the most effective compound in $43 \%(3 / 7)$ of malederived patient tumors, while the other four male tumors were uniquely most sensitive to individual compounds, including BIX01294 (hydrochloride hydrate), HC Toxin, LAQ824, and UNC0631. 


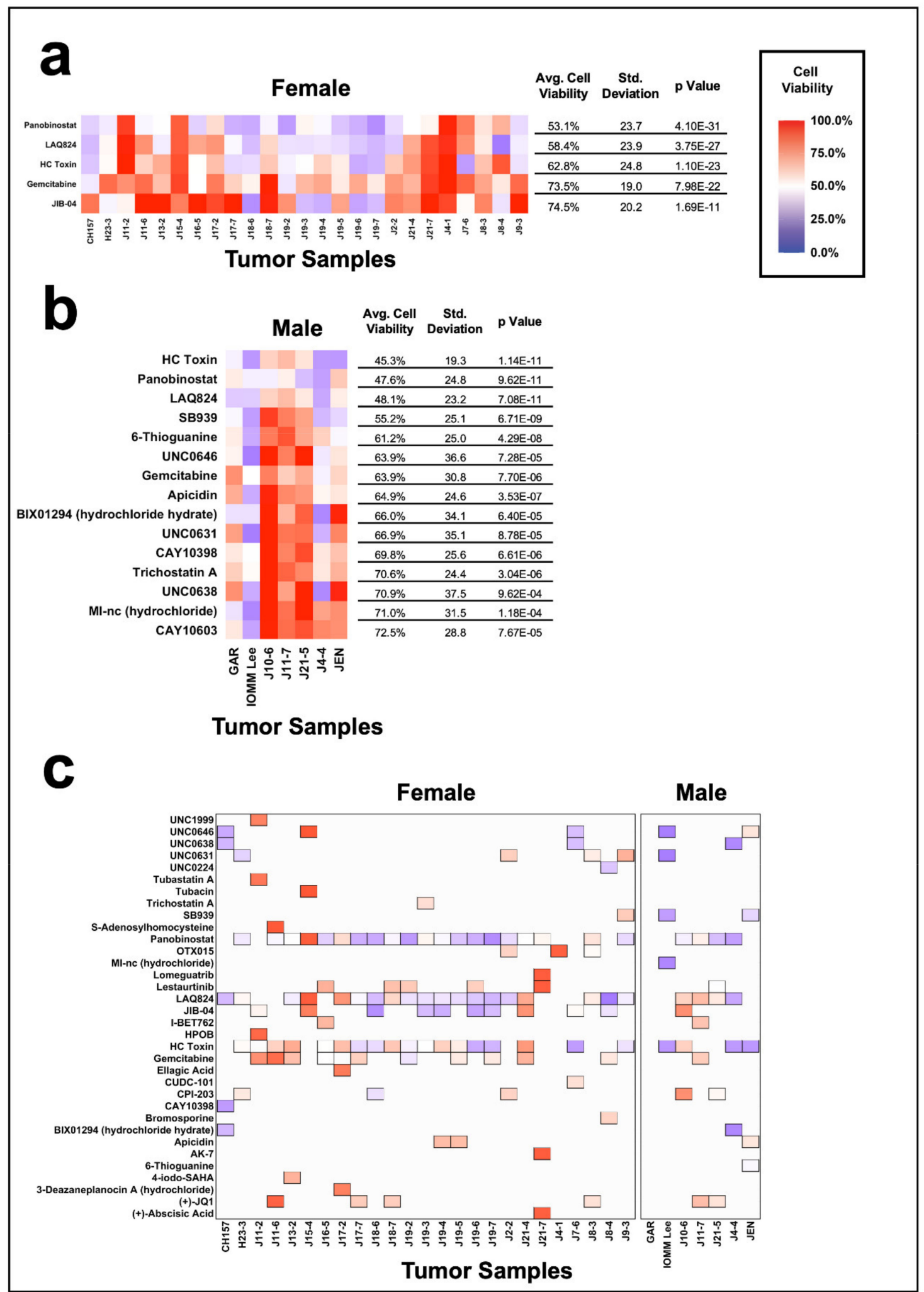

Figure 6. Comparison of the meningioma cohort by patient gender. Heat maps of broadly effective compounds, separated by tumors originating from (a) female patients and (b) male patients. Broadly effective compounds for meningiomas derived from female and male patients are identified as compounds that reduce the average cell viability of the group to $80 \%$ or less and have a $p$ value of less than 0.05 . (c) Heat map of the top five most effective epigenetic compounds, separated by female and male patients. The top five compounds are displayed as filled tiles in the heat map, while compounds that were not in the top five remain as white space. 


\section{Discussion}

In this study, we present a 32-meningioma cohort screened with 139 epigenetic compounds to find targetable mechanisms for the treatment of meningiomas. While all meningiomas displayed a unique sensitivity profile, most were sensitive to a small group of compounds. The most broadly effective compounds identified-panobinostat, LAQ824 and HC toxin - all act via HDAC inhibition. Panobinostat and LAQ824 target class I, class IIa and class IIb HDACs [33,34], while HC-toxin is a reversible, cell permeable HDAC inhibitor $[35,36]$. The two exceptions to this were the single tumor with known prior radiation (Figure 5b), which exhibited greater sensitivity to a Jumanji domain inhibitor, and the single grade 3 tumor (Figure 3c), which showed greater sensitivity to G9a/GLP inhibition, although both of these tumors remained sensitive to HDAC inhibition. These data suggest a common dependence on HDAC function for the viability of meningiomas independent of grade, prior radiation, recurrence, or patient gender; therefore, HDACs present attractive therapeutic targets.

HDACs are an important epigenetic mechanism in meningiomas, in part, because of their regulation of the Akt pathway, which is affected by the inactivation of NF2 [9,11,37]. Previous studies, utilizing meningioma mouse xenograft models, have implicated the efficacy of pan-HDAC inhibitors [38,39]. In a 2013 study, the investigators evaluated panHDAC inhibition with compound AR-42 in Ben-Men cell-line intracranial xenografts and found a significant reduction in tumor growth [38]. In a more recent study, investigators using a patient-derived orthotopic xenograft model achieved a significant reduction in tumor growth using panobinostat [39].

One of the primary limitations of this study was the use of a single dose of drug across a large library of compounds. The reason we limited this study to a single dose was due to the low number of tumor cells available, which were spread across treatments by 139 compounds. In doing so, we were able to ensure our ability to screen the entire library in triplicate for every tumor. The $1 \mathrm{mM}$ dose was selected based on a study by Liston and Davis, which identified $1.47 \mathrm{mM}$ as the median maximum plasma concentration in humans across a cohort of 145 FDA-approved cancer compounds [40]. The limited number of tumor cells also prevented further analysis of the nature of cell death/cytotoxicity versus cytostasis, as we relied on MTS assays to assess drug-driven cell growth inhibition. MTS assays are facile, reproducible, and relatively inexpensive for large-scale screens [32], and we chose this as our primary indicator of drug utility because it is compatible with our culture system.

The total cohort exceeded 30 tumors and therefore was an appropriate size to apply standard population statistics; however, a significant limitation is the lack of population to appropriately power sub-analyses. Nonetheless, this cohort reflects the generalized epidemiologic trends of the disease: in terms of ratios of tumor grades, $80-85 \%$ grade 1 tumors are reported; our cohort was $\sim 79 \%$ grade 1 tumors; $15-20 \%$ grade 2 tumors are reported; our cohort was $\sim 16 \%$ grade $2 ; 1-2 \%$ grade 3 tumors are reported; our single grade 3 tumor would represent $\sim 3 \%[41,42]$. Recurrence rates vary according to tumor grade and extent of resection, but 5-year recurrence rates range from as low as $7 \%$ (grade 1 ) to as high as 78\% (grade 3) [42]. Without subclassification according to patients' previous surgical and clinical parameters, and reflecting our short time course of tumor collection ( $\sim 9$ months), $<10 \%$ of our cohort presented with recurrent tumors, and no individuals with primary tumors within our cohort recurred during the course of this study. Radiation therapy likewise varies as a treatment, mostly for sub-total resections and higher tumor grades, while most grade 1 tumors are not radiated [41,42], making it difficult to generalize. Our cohort had only one patient known to have received radiation and four cases where this was not specified. Female preponderance of the disease is reported to be 2.27:1 [41,42], and our cohort was $\sim 3.5: 1$, female to male. Thus, ratios of tumor grades, female-to-male skew of patients, rates of recurrence, and use radiation therapy among our cohort are remarkably similar to those categories found in larger population studies. We do realize that to achieve sufficient numbers of particular tumor subsets for a more robust statistical 
evaluation (e.g., tumors of higher grade, tumors that are recurrent, or those that have received prior radiation), we will need much longer collection periods and collaborations with other institutions.

The findings of this study present HDAC inhibition as a promising treatment avenue for meningioma. However, as this was a mechanistic screen for classes of epigenetic compounds, further validation studies are still required to identify an ideal clinical candidate compound for further validation in pre-clinical studies. Although the lack of mechanistic studies makes it unclear as to how meningiomas are dependent on HDAC activity, the suppressed growth observed in our in vitro cohort as well as these two in vivo studies [38,39] supports the therapeutic potential of HDAC inhibitors for the treatment of meningioma.

Supplementary Materials: The following are available online at https: / www.mdpi.com/article / 10.3390/jcm10143150/s1, Supplementary Figure S1: Meningioma cohort sensitivity to epigenetic compounds separated by tumor grade. Supplementary Figure S2: Meningioma cohort sensitivity to epigenetic compounds separated by primary and recurrent tumors. Supplementary Figure S3: Meningioma cohort sensitivity to epigenetic compounds separated by history of radiation. Supplementary Figure S4: Meningioma cohort sensitivity to epigenetic compounds separated by patient gender. Supplementary Table S1: Cell viability for each epigenetic compound and meningioma sample. Supplementary Table S2: $p$ value for each epigenetic compound and meningioma sample. Supplementary Table S3: Average cell viability for each epigenetic compound. Supplementary Table S4: The most effective compound for each tumor sample. Supplementary Table S5: Broadly effective epigenetic compounds separated by tumor grade. Supplementary Table S6: Comparison of compound effectiveness across tumor grade. Supplementary Table S7: Broadly effective epigenetic compounds separated by primary and recurrent tumors. Supplementary Table S8: Comparison of compound effectiveness across primary and recurrent tumors. Supplementary Table S9: Broadly effective epigenetic compounds separated by tumor radiation history. Supplementary Table S10: Broadly effective epigenetic compounds separated by patient gender. Supplementary Table S11: Comparison of compound effectiveness between female- and male-derived patient tumors.

Author Contributions: Conceptualization, P.D.T., T.H.W., D.M.D., R.L.J., M.W.G. and D.R.O.; methodology, P.D.T., T.H.W., A.R.F., S.R.S., W.B.F., M.W.G. and D.R.O.; formal analysis, P.D.T., T.H.W., D.M.D., R.L.J., M.W.G. and D.R.O.; resources, D.M.D., K.L., A.S.Y., R.L.J., M.W.G. and D.R.O.; data curation, P.D.T., T.H.W., A.R.F., S.R.S., W.B.F., M.W.G. and D.R.O.; writing-original draft preparation, P.D.T. and T.H.W.; writing-review and editing, P.D.T., T.H.W., D.M.D., K.L., A.S.Y., R.L.J., M.W.G. and D.R.O.; supervision, D.M.D., K.L., A.S.Y., R.L.J., M.W.G. and D.R.O.; project administration, P.D.T., M.W.G. and D.R.O.; funding acquisition, P.D.T., M.W.G. and D.R.O. All authors have read and agreed to the published version of the manuscript.

Funding: This research was funded by the Department of Neurosurgery at the University of Colorado Anschutz and Meningioma Mommas ("Determining the Therapeutic Potential of HDAC Inhibitors in Meningiomas").

Institutional Review Board Statement: This study was approved by the Colorado Multiple Institutional Review Board (COMIRB Protocol 13-3007), with the following approval statement: "This study was reviewed and approved under the "2018 Requirements" of the Federal Policy for the Protection of Human Subjects." Approval date: 14Nov2019; no expiration date. The study was conducted according to the guidelines of the Declaration of Helsinki and approved by the Institutional Review Board (or Ethics Committee) of the University of Colorado Anschutz (COMIRB Protocol \#13-3007; approval date, 14 November 2019).

Informed Consent Statement: Informed consent was obtained from all subjects involved in the study. Study subjects are de-identified.

Data Availability Statement: Data are contained within the article or supplementary material; additional requests for information should be directed to the corresponding authors.

Acknowledgments: The authors particularly wish to thank the University of Colorado Neurosurgery Nervous System Biorepository for acquisition of, and access to, the tumor materials; to the neurosurgeons who contributed the tumor samples; and to the patients who selflessly consented to the study and donated their tissues. 
Conflicts of Interest: The authors declare no conflict of interest. The funders had no role in the design of the study; in the collection, analyses, or interpretation of data; in the writing of the manuscript, or in the decision to publish the results.

\section{References}

1. Ostrom, Q.T.; Gittleman, H.; Truitt, G.; Boscia, A.; Kruchko, C.; Barnholtz-Sloan, J.S. Cbtrus statistical report: Primary brain and other central nervous system tumors diagnosed in the united states in 2011-2015. Neuro-Oncology 2018, 20, iv1-iv86. [CrossRef]

2. Choy, W.; Kim, W.; Nagasawa, D.; Stramotas, S.; Yew, A.; Gopen, Q.; Parsa, A.T.; Yang, I. The molecular genetics and tumor pathogenesis of meningiomas and the future directions of meningioma treatments. Neurosurg. Focus 2011, 30, E6. [CrossRef]

3. Rogers, L.; Barani, I.; Chamberlain, M.; Kaley, T.J.; McDermott, M.; Raizer, J.; Schiff, D.; Weber, D.C.; Wen, P.Y.; Vogelbaum, M.A. Meningiomas: Knowledge base, treatment outcomes, and uncertainties. A Rano Review. J. Neurosurg. 2015, 122, 4-23. [CrossRef]

4. Aghi, M.K.; Carter, B.S.; Cosgrove, G.R.; Ojemann, R.G.; Amin-Hanjani, S.; Martuza, R.L.; Curry, W.T., Jr.; Barker, F.G., 2nd. Long-term recurrence rates of atypical meningiomas after gross total resection with or without postoperative adjuvant radiation. Neurosurgery 2009, 64, 56-60, discussion 60. [CrossRef]

5. Jaaskelainen, J. Seemingly complete removal of histologically benign intracranial meningioma: Late recurrence rate and factors predicting recurrence in 657 patients. A multivariate analysis. Surg. Neurol. 1986, 26, 461-469. [CrossRef]

6. Van Alkemade, H.; de Leau, M.; Dieleman, E.M.; Kardaun, J.W.; van Os, R.; Vandertop, W.P.; van Furth, W.R.; Stalpers, L.J. Impaired survival and long-term neurological problems in benign meningioma. Neuro-Oncology 2012, 14, 658-666. [CrossRef]

7. Nigim, F.; Wakimoto, H.; Kasper, E.M.; Ackermans, L.; Temel, Y. Emerging medical treatments for meningioma in the molecular era. Biomedicines 2018, 6, 86. [CrossRef]

8. Preusser, M.; Brastianos, P.K.; Mawrin, C. Advances in meningioma genetics: Novel therapeutic opportunities. Nat. Rev. Neurol. 2018, 14, 106-115. [CrossRef] [PubMed]

9. Petrilli, A.M.; Fernandez-Valle, C. Role of merlin/nf2 inactivation in tumor biology. Oncogene 2016, 35, 537-548. [CrossRef]

10. Riemenschneider, M.J.; Perry, A.; Reifenberger, G. Histological classification and molecular genetics of meningiomas. Lancet Neurol. 2006, 5, 1045-1054. [CrossRef]

11. Clark, V.E.; Erson-Omay, E.Z.; Serin, A.; Yin, J.; Cotney, J.; Ozduman, K.; Avsar, T.; Li, J.; Murray, P.B.; Henegariu, O.; et al. Genomic analysis of non-nf2 meningiomas reveals mutations in traf7, klf4, akt1, and smo. Science 2013, 339, 1077-1080. [CrossRef]

12. Brastianos, P.K.; Horowitz, P.M.; Santagata, S.; Jones, R.T.; McKenna, A.; Getz, G.; Ligon, K.L.; Palescandolo, E.; Van Hummelen, P.; Ducar, M.D.; et al. Genomic sequencing of meningiomas identifies oncogenic smo and akt1 mutations. Nat. Genet. 2013, 45, 285-289. [CrossRef] [PubMed]

13. Clark, V.E.; Harmanci, A.S.; Bai, H.; Youngblood, M.W.; Lee, T.I.; Baranoski, J.F.; Ercan-Sencicek, A.G.; Abraham, B.J.; Weintraub, A.S.; Hnisz, D.; et al. Recurrent somatic mutations in polr2a define a distinct subset of meningiomas. Nat. Genet. 2016, 48, 1253-1259. [CrossRef]

14. Shin, I.; Park, Y.W.; Ahn, S.S.; Kang, S.G.; Chang, J.H.; Kim, S.H.; Lee, S.K. Clinical and diffusion parameters may noninvasively predict tert promoter mutation status in grade ii meningiomas. J. Neuroradiol. 2021. [CrossRef]

15. Barresi, V.; Simbolo, M.; Fioravanzo, A.; Piredda, M.L.; Caffo, M.; Ghimenton, C.; Pinna, G.; Longhi, M.; Nicolato, A.; Scarpa, A. Molecular profiling of 22 primary atypical meningiomas shows the prognostic significance of $18 \mathrm{q}$ heterozygous loss and cdkn2a/b homozygous deletion on recurrence-free survival. Cancers 2021, 13, 903. [CrossRef] [PubMed]

16. Biczok, A.; Kraus, T.; Suchorska, B.; Terpolilli, N.A.; Thorsteinsdottir, J.; Giese, A.; Tonn, J.C.; Schichor, C. Tert promoter mutation is associated with worse prognosis in who grade ii and iii meningiomas. J. Neurooncol. 2018, 139, 671-678. [CrossRef]

17. Juratli, T.A.; Thiede, C.; Koerner, M.V.A.; Tummala, S.S.; Daubner, D.; Shankar, G.M.; Williams, E.A.; Martinez-Lage, M.; Soucek, S.; Robel, K.; et al. Intratumoral heterogeneity and tert promoter mutations in progressive/higher-grade meningiomas. Oncotarget 2017, 8, 109228-109237. [CrossRef]

18. Guyot, A.; Duchesne, M.; Robert, S.; Lia, A.S.; Derouault, P.; Scaon, E.; Lemnos, L.; Salle, H.; Durand, K.; Labrousse, F. Analysis of cdkn2a gene alterations in recurrent and non-recurrent meningioma. J. Neurooncol. 2019, 145, 449-459. [CrossRef]

19. Hatada, I. The epigenomics of cancer. In An Omics Perspective on Cancer Research; Cho, W.C., Ed.; Springer: New York, NY, USA, 2010; pp. 51-67.

20. Perdigoto, C.N. Epigenetic cancer evolution, one cell at a time. Nat. Rev. Genet. 2019, 20, 434-435. [CrossRef]

21. Esteller, M. Epigenetics in cancer. N. Engl. J. Med. 2008, 358, 1148-1159. [CrossRef]

22. Gillette, T.G.; Hill, J.A. Readers, writers, and erasers: Chromatin as the whiteboard of heart disease. Circ. Res. 2015, 116, 1245-1253. [CrossRef]

23. Al-Mefty, O.; Kadri, P.A.; Pravdenkova, S.; Sawyer, J.R.; Stangeby, C.; Husain, M. Malignant progression in meningioma: Documentation of a series and analysis of cytogenetic findings. J. Neurosurg. 2004, 101, 210-218. [CrossRef] [PubMed]

24. Barski, D.; Wolter, M.; Reifenberger, G.; Riemenschneider, M.J. Hypermethylation and transcriptional downregulation of the timp3 gene is associated with allelic loss on 22q123 and malignancy in meningiomas. Brain Pathol. 2010, 20, 623-631. [CrossRef] [PubMed]

25. Bello, M.J.; Aminoso, C.; Lopez-Marin, I.; Arjona, D.; Gonzalez-Gomez, P.; Alonso, M.E.; Lomas, J.; de Campos, J.M.; Kusak, M.E.; Vaquero, J.; et al. DNA methylation of multiple promoter-associated cpg islands in meningiomas: Relationship with the allelic status at 1p and 22q. Acta Neuropathol. 2004, 108, 413-421. [CrossRef] [PubMed] 
26. Berman, B.P.; Weisenberger, D.J.; Aman, J.F.; Hinoue, T.; Ramjan, Z.; Liu, Y.; Noushmehr, H.; Lange, C.P.; van Dijk, C.M.; Tollenaar, R.A.; et al. Regions of focal DNA hypermethylation and long-range hypomethylation in colorectal cancer coincide with nuclear lamina-associated domains. Nat. Genet. 2011, 44, 40-46. [CrossRef]

27. He, S.; Pham, M.H.; Pease, M.; Zada, G.; Giannotta, S.L.; Wang, K.; Mack, W.J. A review of epigenetic and gene expression alterations associated with intracranial meningiomas. Neurosurg. Focus 2013, 35, E5. [CrossRef]

28. Bush, M.L.; Oblinger, J.; Brendel, V.; Santarelli, G.; Huang, J.; Akhmametyeva, E.M.; Burns, S.S.; Wheeler, J.; Davis, J.; Yates, C.W.; et al. Ar42, a novel histone deacetylase inhibitor, as a potential therapy for vestibular schwannomas and meningiomas. Neuro-Oncology 2011, 13, 983-999. [CrossRef] [PubMed]

29. Chen, C.S.; Weng, S.C.; Tseng, P.H.; Lin, H.P.; Chen, C.S. Histone acetylation-independent effect of histone deacetylase inhibitors on akt through the reshuffling of protein phosphatase 1 complexes. J. Biol. Chem. 2005, 280, 38879-38887. [CrossRef] [PubMed]

30. Rong, R.; Tang, X.; Gutmann, D.H.; Ye, K. Neurofibromatosis 2 (nf2) tumor suppressor merlin inhibits phosphatidylinositol 3-kinase through binding to pike-l. Proc. Natl. Acad. Sci. USA 2004, 101, 18200-18205. [CrossRef]

31. DeQuach, J.A.; Yuan, S.H.; Goldstein, L.S.; Christman, K.L. Decellularized porcine brain matrix for cell culture and tissue engineering scaffolds. Tissue Eng. Part A 2011, 17, 2583-2592. [CrossRef]

32. Riss, T.L.; Moravec, R.A.; Niles, A.L.; Duellman, S.; Benink, H.A.; Worzella, T.J.; Minor, L. Cell viability assays. In Assay Guidance Manual; Markossian, S., Sittampalam, G.S., Grossman, A., Brimacombe, K., Arkin, M., Auld, D., Austin, C.P., Baell, J., Caaveiro, J.M.M., Chung, T.D.Y., et al., Eds.; Eli Lilly \& Company and the National Center for Advancing Translational Sciences: Bethesda, MD, USA, 2004. [PubMed]

33. Thomas, S.; Miller, A.; Thurn, K.T.; Munster, P. Chapter 37—clinical applications of histone deacetylase inhibitors. In Handbook of Epigenetics; Tollefsbol, T., Ed.; Academic Press: San Diego, CA, USA, 2011; pp. 597-615.

34. Jin, J.X.; Lee, S.; Taweechaipaisankul, A.; Kim, G.A.; Lee, B.C. The hdac inhibitor laq824 enhances epigenetic reprogramming and in vitro development of porcine scnt embryos. Cell Physiol. Biochem. 2017, 41, 1255-1266. [CrossRef]

35. Brosch, G.; Ransom, R.; Lechner, T.; Walton, J.D.; Loidl, P. Inhibition of maize histone deacetylases by hc toxin, the host-selective toxin of cochliobolus carbonum. Plant Cell 1995, 7, 1941-1950.

36. Darkin-Rattray, S.J.; Gurnett, A.M.; Myers, R.W.; Dulski, P.M.; Crumley, T.M.; Allocco, J.J.; Cannova, C.; Meinke, P.T.; Colletti, S.L.; Bednarek, M.A.; et al. Apicidin: A novel antiprotozoal agent that inhibits parasite histone deacetylase. Proc. Natl. Acad. Sci. USA 1996, 93, 13143-13147. [CrossRef] [PubMed]

37. Goutagny, S.; Kalamarides, M. Meningiomas and neurofibromatosis. J. Neurooncol. 2010, 99, 341-347. [CrossRef] [PubMed]

38. Burns, S.S.; Akhmametyeva, E.M.; Oblinger, J.L.; Bush, M.L.; Huang, J.; Senner, V.; Chen, C.S.; Jacob, A.; Welling, D.B.; Chang, L.S. Histone deacetylase inhibitor ar-42 differentially affects cell-cycle transit in meningeal and meningioma cells, potently inhibiting nf2-deficient meningioma growth. Cancer Res. 2013, 73, 792-803. [CrossRef] [PubMed]

39. Zhang, H.; Qi, L.; Du, Y.; Huang, L.F.; Braun, F.K.; Kogiso, M.; Zhao, Y.; Li, C.; Lindsay, H.; Zhao, S.; et al. Patient-derived orthotopic xenograft (pdox) mouse models of primary and recurrent meningioma. Cancers 2020, 12, 1478. [CrossRef]

40. Liston, D.R.; Davis, M. Clinically relevant concentrations of anticancer drugs: A guide for nonclinical studies. Clin. Cancer Res. 2017, 23, 3489-3498. [CrossRef]

41. Buerki, R.A.; Horbinski, C.M.; Kruser, T.; Horowitz, P.M.; James, C.D.; Lukas, R.V. An overview of meningiomas. Future Oncol. 2018, 14, 2161-2177. [CrossRef]

42. Ogasawara, C.; Philbrick, B.D.; Adamson, D.C. Meningioma: A review of epidemiology, pathology, diagnosis, treatment, and future directions. Biomedicines 2021, 9, 319. [CrossRef] 